\title{
Queueing model analysis of shopping malls in COVID-19 pandemic era: a case study
}

\author{
Aymen M. Al-Kadhimi*, Mustafa Abdulkadhim and Salim A. Mohammed Ali \\ College of Information Engineering, Al-Nahrain University, Baghdad, Iraq
}

Received: 26-August-2021; Revised: 15-December-2021; Accepted: 17-December-2021

(C)2021 Aymen M. Al-Kadhimi et al. This is an open access article distributed under the Creative Commons Attribution (CC BY) License, which permits unrestricted use, distribution, and reproduction in any medium, provided the original work is properly cited.

\begin{abstract}
Queueing forms a daily routine of our lives wherever there are limitations in resources with competition. The ongoing Coronavirus Disease of 2019 (COVID-19) pandemic undeniably has its impacts on people's daily life. That in turn affected the number of customers who used to go outside for different outdoor activities. In this paper, a shopping mall is considered as a case study to propose an appropriate queueing model within these current pandemic circumstances. The proposed model consists of an open network with 10 nodes represent different real-existing serving stations. The queueing model is first analysed to check its stability that is evident analytically on the long run. The performance of the proposed network model is investigated using different measures. Based on recorded arrival and service rates, the mathematical derivations show that the traffic intensity of the system is still below 1, hence ensures stability. Also, the average number of customers in the network is 16.76 customers with only 2.66 customers awaiting in queues. Furthermore, the mean time a customer spends in the queue is only 0.16 min from a total spend on the network about 1.006 min. This indicates that the majority of time customers spend is in service with a very short waiting time due to current COVID-19 pandemic consequences, and the waiting time and queue lengths will undoubtedly increase once normal social life resumes.
\end{abstract}

\section{Keywords}

Steady state, Arrival rate, Service rate, Queue length, Waiting time, Idle state, Shopping mall, COVID-19.

\section{Introduction}

In single-node queueing systems, customers arrive at a service station to receive a single service and then depart with never to return, whereas in multiple-node systems, customers require services at more than one node/station for one or many times [1]. Those multiple-node systems can be referred as a network of nodes where each node acts as a service station that has a storage place for queues with the possibility of deploying multiple servers for handling customer's requests. For examples, hospitals or doctor's office, manufacturing, packets entering a network of routers, etc. Also, those multiple-node network queues may be experienced lined up for instance, in shopping malls that may aggregate supermarket checkout lanes, retail clothing outlets cashiers, flight booking offices or restaurants.

*Author for correspondence

1545
Furthermore, queueing models and theory have witnessed extensive applicability in telecommunications networks in general [1,2], and particularly wide implementation in cognitive radio networks where prevalent queueing models act as priority models, and vacation models in which a server is going on vacation and cease to offer service for a time period [3].

To design a queueing network, the key elements (customers and servers) are pre-addressed. Those elements in the case of designing queueing models for shopping malls are the shoppers as customers and checkout stations as servers. In addition, the arrival and services probability distributions are considered. Even though there are several queueing models classified whether the distribution is Markovian (i.e., exponential/ Poisson), deterministic, Erlang, hyperexponential or general, the Markovian models are the wide realistic ones deployed practically [4]. Furthermore, among different queueing network types, open Jackson network is the most convenient choice for developing a queueing model for shopping 
malls. That is because it describes a network of several serving nodes at which customers arrive to from outside with Poisson distribution at a certain arrival rate to be processed at a reasonable service rate [5]. Once customers/ shoppers are served at particular nodes, then they can get other services at different nodes facilities before departing the network. Consequently, queueing systems should be examined to check its stability, which means measuring how the whole network of nodes is able to handle customers who ask for service without ending with increasing waiting time which leads to system failure. Moreover, the average queue length, average number of customers in the network, mean system time and mean serving time are all essential measures that determine designed queueing system performance [1].

The motivation behind the work of this paper is to propose a network queueing system of a shopping mall along with its mathematical modelling for stability examination and performance evaluation. Furthermore, highlighting the impacts of Coronavirus Disease of 2019 (COVID-19) pandemic on the customers population of shopping malls is crucial. That is because once the queueing system is mathematically analyzed, each of network nodes is determined whether it is in a marginally critical situation or stable. For those critical nodes with current ongoing intensity of shoppers, it will not be the case if the normal life resumes as the population of shoppers will increase. In consequence, the number of servers (checkouts) can be re-considered for those particular nodes, and similarly for the stable nodes with light customers intensity can be scalable. Hence, system failure can be overcome by optimizing the system analytically. The system model in this paper is developed for the Baghdad shopping mall, one of the largest shopping malls in the Iraqi's capital. The performance of the proposed system is derived and analyzed based on different measures such as node utilization, mean queue length, idle steady state probability and customer mean waiting time. Although the model is proposed and developed for the Baghdad mall as a case study, the design workflow analysis can be generalized to suit other similar life scenarios such as other malls, supermarkets or clinics, and that is one of the main objectives of this paper.

The rest of the paper is organized as follows. Section 2 involves some recent related works in literature. Afterwards, the design assumptions and the proposed state transition diagram along with a definition of each network node in terms of queue type are all provided is section 3 . Then, the system analysis is analytically investigated that includes system stability check, nodes utilization determination and steady state probability derivations. Section 4 discusses the results of the proposed system to evaluate its performance. Afterwards, section 5 presents an overall analysis and comparative discussion of performance of the designed system with addressing some of its limitations. Lastly, some future works and conclusions are drawn in section 6.

\section{Literature review}

In literature, many researchers have evaluated and analyzed queueing models for supermarkets. Jhala and Bhathawala in [6] presented a mathematical queueing model for a supermarket in which they estimated the number of servers required to ensure minimum waiting time and total cost optimization along with direct customer satisfaction.

Igwe et al. [7] studied the efficiency of queueing management in supermarkets in Nigeria. They presented an analysis in the queue management in the Makurdi town in which suggestions are made to assist mitigate queuing problems in the town that many towns in developing countries facing similar challenges too.

Priyangika and Cooray [8] analyzed in their paper the supermarket checkout operations based on the empirical data of service units by means of queuing theory.

Prasad et al. [9] presented a mathematical modelling and a comparison study of two queueing models: single-queue multi-server and multi-queue multiserver. They have proven in the study that the mean total cost of single-queue multi-server is less, for their model, than multi-queue multi-server.

Chinwuko et al. [10] studied the analysis of a bank in which they suggested a $40 \%$ increase in the number of servers so that the queueing system become more stable with a reasonable customer waiting time.

Sharma and Barua [11] developed in their paper a queueing model for a grocery store to eliminate congestion. Also, they proposed an alternative model other than the existing one and validated the recorded data that were collected over two weeks on different grocery's working hours. Moreover, queueing theory has been analysed recently in healthcare sections to cope with COVID-19 pandemic. 
Majedkan et al. [12] investigated the queue modelling of diagnosing the arrivals to Duhok Iraqi city to determine the expected and real waiting times. The Analysis was based on 7-days data collection by a healthcare staff. The results showed that each arrival might be standing in the queue for almost 32 minutes to get diagnosed. Also, the study model estimated the waiting time to provide knowledge to the medical teams about the number of clients awaiting in queues and how many of them will leave away per day.

Chatterjee et al. [13] presented a stochastic modelling for COVID-19 pandemic for assessment of different healthcare services offered by available sources. Also, the study estimated the impacts of certain nonpharmacological interventions on the epidemic such as quarantine and social distancing.

Wang [14] developed a novel computing technique for investigating the process of health inspection depending on management methodology of threecolour risk screening applied at check points that discriminate borders. He concentrated on the perspective recovery of the epidemic in terms of economy with presenting a queueing system for health channels and analysing the process of health inspection.

Rezzouki et al. [15] proposed a system that offers monitoring of customers standing in queue with onemeter as social distancing. They applied an array sensor based on infrared and developed an algorithm to ensure customer's privacy preservation and reduced energy consumption.

Tyagi et al. [16] applied queueing theory for the purpose of boosting the server utilization of an ophthalmic hospital. The study showed that the server utilization of the patient's registration has increased from 0.63 to 1.21 . As a result, they compared the records of patients' registrations after one year of applying the model which proven higher server utilization with less length of waiting queues.

In the same year, Jen et al. [17] developed a queue model approach that investigated the capacity of medical hospitality and Intensive Care Unit (ICU) during COVID-19 pandemic. Based on statistical assessed data of Lombardy, Italy, they evaluated the situation of hospitals' capacity in terms of ICU beds and isolated units by using their developed queueing model for monitoring. Moreover, several studies have dealt with queues in medical centres via presenting applications for queue management systems.

A study was presented by Thirupathieswaran et al. [18] to investigate the benefit of applying a queue maintenance system to ensure minimized queues at hospitals using a smart mobile application. Their proposed system provides the patients with managing appointments and maintaining medical records in a way that avoids visitors the queueing waiting time during the pandemic period.

Similarly, Soman et al. [19] proposed a smart management system for queues in hospitals that is mobile-augmented based. They showed that the system is configurable and integrable with information systems of medical centres via providing smart algorithms for the generation of tokens among patients.

Even though the aforementioned studies have proven their valuable contributions, there is still a gap to be filled in terms of future analytical countermeasures of the era after pandemic. Unlike the statistical data based and simulation-based studies, this paper presents an analytical based study of a queueing system design that can be generalized for developing other similar queueing scenarios. This approach can be employed to countermeasure the post-pandemic era by mathematically evaluating the queueing system of a shopping mall in current ongoingpandemic period. The queueing system nodes are topologized along with the derivation of their steady state probabilities. The study can be utilized as a guide to monitor the critical system nodes that barely can handle present customers intensity, and then replan (e.g., increasing the checkout stations) those nodes so that the system performance does not degrade when the normal person's life resumes after pandemic.

\section{Methods}

3.1System design methods

The system design is proposed in three sequential phases, namely design assumptions, design topology\& data recording and design analysis and performance analysis. The workflow diagram of the phases is shown is Figure 1. Firstly, in pahse1, several design assumptions are presented to suit the proposed system of the mall specifically as the case study. Instances of these assumptions are: Jackson network with Markovian Poisson arrivals, exponentially distributed service rates, number of network nodes, scheduling discipline and customer 
behaviours. All the assumptions are listed in this section then. Afterwards, phase 2 emerges where the topology of the system (network) is proposed with all particular transition probabilities among nodes that fit the mall's status. Also, the arrival and service rates of each individual node are recorded by human observations. These first two phases, except data records, are presented in this section. Once all network setups are prepared, it is the time for the phase 3 to be involved. This last phase includes both system mathematical analysis and performance measures which are investigated in Section 4 and Section 5 respectively. Section 6 then presents an overall analysis and comparative discussion of performance for the designed system. The three phases are proposed and analysed in this paper to provide a unique analytical guide to check the system stability in terms of the mall's customers handling along with the possibility of future scalability expansion in tolerant nodes.

The following assumptions are considered in phase 1 before diving into the mathematical analysis.

- The overall system consists of a network of 10 nodes denoted as N1, N2, ...N10.

- The system's nodes represent each of entrance gates, fast-food restaurants, hypermarket, clothing retail outlets, exchange offices, a corridor and exits.

- Open network: two nodes that customers arrive from as external entrance sources and there are two nodes from which customers depart the system.

- The number of customers (k) at any node is independent of the number of customers at every other node: $\pi(k)=\pi_{1}\left(k_{1}\right) \cdot \pi_{2}\left(k_{2}\right) \ldots \pi_{10}\left(k_{10}\right)$

- Jackson network with the individual nodes fed by Poisson arrivals with rate $\lambda$ customer/ minute.

- Markovian exponentially distributed service time with service rate equal to $\mu$ customer/ minute.

- The arrivals via the pedestrian entrance must depart from the pedestrian exit. Also, the arrivals via the parking entrance must depart from the parking exit.

- There is neither unusual customer behaviour (join the queue and wait till served) nor unusual server behaviour.

- The mean arrival rate is less than the mean service rate (i.e., $\lambda<\mu$ ).

- Due to the ongoing COVID-19 pandemic, it is noticeable that the number of customers of the mall is generally limited. Also, most of the mall's retail outlets are not fully loaded with people nowadays. In consequence, the system is assumed to be infinite population and infinite capacity.

- The scheduling discipline is First Come First Served (FCFS) for all nodes in the system.

After defining the design assumptions, a general system description is presented in terms of particular notations of the ten network nodes. The system is sub-divided into different kind of service nodes. The representation of each network node (N1 to N10) is shown in Table 1. N1 and N2 represent the main arrival entrances to the mall for pedestrian customers and those who get in by cars. Similarly, N9 and N10 represent the pedestrians and parking exits respectively. The mall's serving facilities: restaurants, hypermarket, clothing retails and exchange offices are denoted by N4, N5, N6 and N7 respectively. $\mathrm{N} 3$ is placed so that it aggregates all shoppers via a single node for ease of analysis. N8 has been effectively proposed in the design to act as the mall corridor so that it interconnects all facilities together, otherwise the mathematical analysis becomes complex when representing transitions among facility nodes directly. On the other hand, the nodes have a different queue model depending on the available servers involved. Table 2 depicts each node with its queue model type. The number of servers describes the number of checkout stations and that are variations among nodes as indicated in the comments of Table 2. N3 and N8 are assumed to have an infinite number of servers since they represent intermediate hall/ corridor nodes with many walking lanes that add delay to the network. Similarly, N9 is considered as a delay node since it serves pedestrian shoppers who depart with sufficient number of exit lanes.

Table 1 Nodes representations

\begin{tabular}{ll}
\hline Node & Representation \\
\hline N1 & Pedestrian entrance \\
\hline N2 & Parking entrance \\
\hline N3 & Mall arrivals \\
\hline N4 & Fast-food restaurants \\
\hline N5 & The Cosmo hypermarket \\
\hline N6 & Clothing retail outlets \\
\hline N7 & Exchange offices \\
\hline N8 & Corridor \\
\hline N9 & Pedestrian exit \\
\hline N10 & Car exit \\
\hline
\end{tabular}




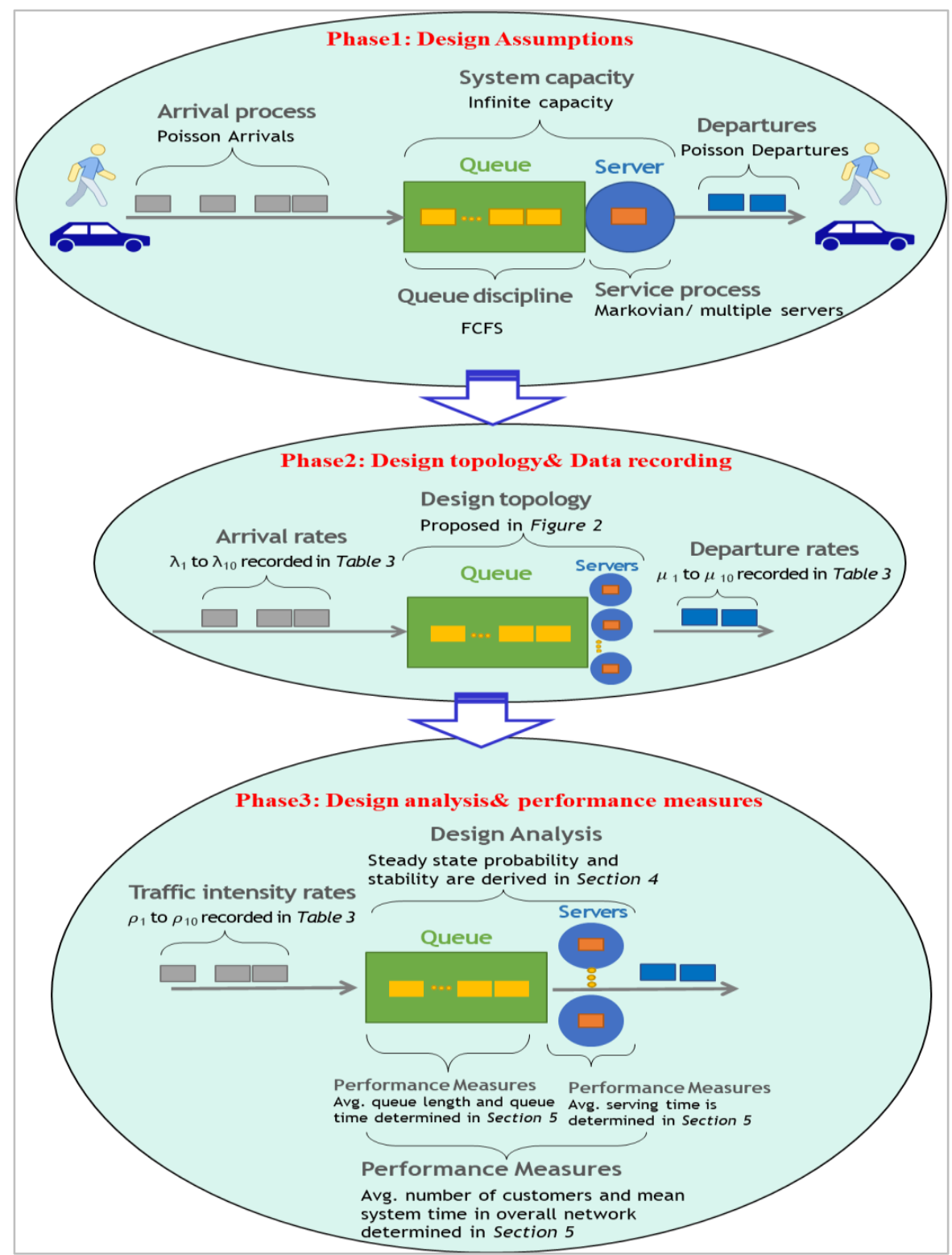

Figure 1 The system design flow phases

The structural network topology is crucial in the analysis as it describes the allowable transitions among service centres. The overall state diagram of the proposed system is shown in Figure 2. The probabilities of the nodes are affixed of the diagram's transitions. The one the arrivals gather into N3, they choose whether they intend to visit N4, N5, N6 or N7 depends on the service they wish to get. After a customer gets their service, they may wish to get another kind of service (for example, going to the supermarket after having a fast meal). In this case, the importance of $\mathrm{N} 8$ comes to the stage, which interconnects the four upper nodes so that they can go back and forth between different kind of services. Afterwords, the customers exit from the system via either the main pedestrian exit or through the car exit. The customers who do not intend to buy anything but roaming in the mall should jump from $\mathrm{N} 3$ to $\mathrm{N} 8$ to depart the system either via N9 or N10. This category of customers occupies the least probability at 0.05 compared with those who get served by fast-food restaurants. Also, it is noticeable that the customers who intend to have meals once they enter the mall through N3 to N4 is with probability of 0.45 , and that is more than those who prefer to get their food after being served by N5, N6 or N7 with a probability of 
0.3. Bearing in mind the assumption that is previously highlighted that the arrivals via the pedestrian entrance must depart later from the pedestrian exit (i.e., $\lambda_{\text {in } 1}=\lambda_{\text {out } 1}$ ). Similarly, the arrivals via the parking entrance must depart later from the parking exit (i.e., $\lambda_{\text {in } 2}=\lambda_{\text {out } 2}$ ). These later two conditions are assumed intuitively since the logical flow of the people who ride their cars into the mall is to depart it using the same mean and that the case also for pedestrians.

Table 2 Nodes queueing types

\begin{tabular}{|c|c|c|}
\hline Node & Queue model & Comment \\
\hline $\mathrm{N} 1$ & $\mathrm{M} / \mathrm{M} / 4$ & $\begin{array}{l}4 \text { checkpoints: } 2 \text { on men's } \\
\text { entrance }+2 \text { on the women's } \\
\text { side }\end{array}$ \\
\hline $\mathrm{N} 2$ & $\mathrm{M} / \mathrm{M} / 1$ & 1 parking entrance checkpoint \\
\hline N3 & $\mathrm{M} / \mathrm{M} / \infty$ & Delay node \\
\hline N4 & $\mathrm{M} / \mathrm{M} / 20$ & 20 restaurants: 1 cashier each \\
\hline N5 & $\mathrm{M} / \mathrm{M} / 6$ & 6 checkout lanes \\
\hline N6 & $\mathrm{M} / \mathrm{M} / 25$ & 25 retailers: 1 cashier each \\
\hline N7 & $\mathrm{M} / \mathrm{M} / 14$ & $\begin{array}{l}14 \text { servers: for money transfer } \\
+ \text { customer service }\end{array}$ \\
\hline N8 & $\mathrm{M} / \mathrm{M} / \infty$ & Delay node \\
\hline N9 & $\mathrm{M} / \mathrm{M} / \infty$ & Infinite number of servers \\
\hline N10 & $\mathrm{M} / \mathrm{M} / 1$ & 1 parking exit checkpoint \\
\hline
\end{tabular}

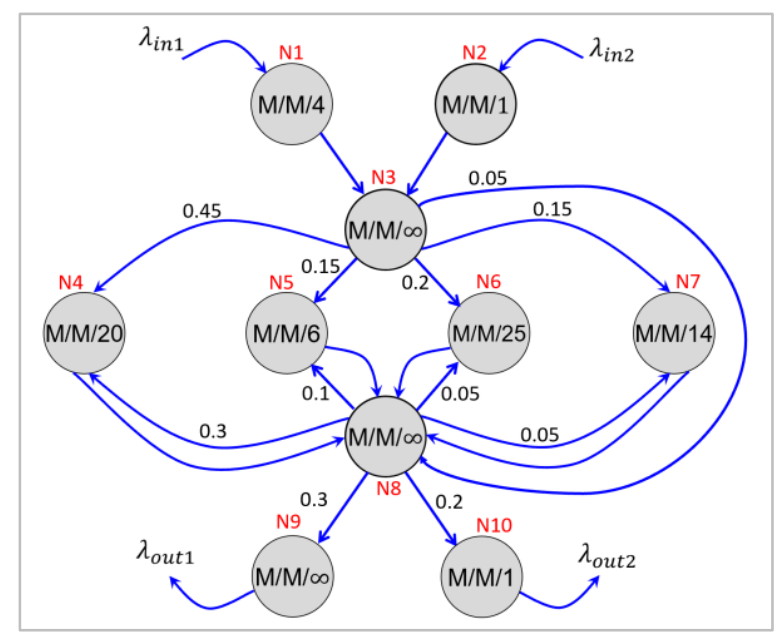

Figure 2 The proposed state transition diagram

\subsection{System analysis}

Prior to dealing with the mathematical analysis of the system model, the data are recorded in customers/minute for both the arrival rate $\lambda_{\text {in }}$ and the service rates $\mu_{i}$ for $i=1,2,3, \ldots, 10$ where $i$ is the node number. The external arrivals $\lambda_{\text {in }}$ and $\mu_{1}$ to $\mu_{10}$ data are record by observation whereas $\lambda_{3}$ to $\lambda_{10}$ are derived and calculated as below. Those data are presented in Table 3. Bearing in mind these readings are subjected to current ongoing COVID-19 pandemic consequences. In other words, the values of $\lambda_{i}$ and $\mu_{i}$ will definitely alter in the future if normal people life resumes.

Now, calculations for arrival rate per node, $\lambda_{i}$ for $i=1,2,3, \ldots, 10$ where $i$ is the node number, are determined as below (Equation 1 to 10).

$\lambda_{1}=\lambda_{\text {in } 1}$

$\lambda_{2}=\lambda_{\text {in } 2}=\frac{2}{3} \lambda_{\text {in } 1}$

$\lambda_{3}=\lambda_{\text {in } 1}+\lambda_{\text {in } 2}=\frac{5}{3} \lambda_{\text {in } 1}$

$\lambda_{3}=\lambda_{i n 1}+\lambda_{i n 2}=\frac{5}{3} \lambda_{i n 1}$

$\lambda_{4}=0.45 \lambda_{3}+0.3 \lambda_{8}=\frac{3}{4} \lambda_{\text {in } 1}+0.3 \lambda_{8}$

$\lambda_{5}=0.15 \lambda_{3}+0.1 \lambda_{8}=\frac{1}{4} \lambda_{\text {in } 1}+0.1 \lambda_{8}$

$\lambda_{6}=0.2 \lambda_{3}+0.05 \lambda_{8}=\frac{1}{3} \lambda_{\text {in } 1}+0.05 \lambda_{8}$

$\lambda_{7}=0.15 \lambda_{3}+0.05 \lambda_{8}=\frac{1}{4} \lambda_{\text {in } 1}+0.05 \lambda_{8}$

$\lambda_{8}=0.05 \lambda_{3}+\lambda_{4}+\lambda_{5}+\lambda_{6}+\lambda_{7}$

$\lambda_{9}=\lambda_{\text {out } 1}=0.3 \lambda_{8}$

$\lambda_{10}=\lambda_{\text {out } 2}=0.2 \lambda_{8}$

where $\lambda_{\text {out } 1}$ and $\lambda_{\text {out } 2}$ are the rates of customers who depart from pedestrian and car exits respectively.

We substitute Equation 3 to 7 in Equation 8 to get:

$$
\begin{aligned}
\lambda_{8}=\frac{1}{12} \lambda_{i n 1}+\frac{3}{4} & \lambda_{i n 1}+0.3 \lambda_{8}+\frac{1}{4} \lambda_{i n 1}+0.1 \lambda_{8} \\
& +\frac{1}{3} \lambda_{i n 1}+0.05 \lambda_{8}+\frac{1}{4} \lambda_{i n 1} \\
& +0.05 \lambda_{8}
\end{aligned}
$$

$\lambda_{8}=\frac{5}{3} \lambda_{\text {in } 1}+0.5 \lambda_{8} \rightarrow \lambda_{8}=\frac{10}{3} \lambda_{\text {in } 1}$

We substitute Equation 11 in Equation 4 to 10 along with $\lambda_{i n 1}=10$ customer/ minute, to get the arrival rates per each node as depicted in Table 3 .

Now to evaluate the model and check its stability, the traffic intensities are determined as below (Equation 12).

$\rho_{i}=\frac{\lambda_{i}}{c_{i} \mu_{i}}$ for $i=0,1,2, \ldots 10$

where $c_{i}$ represents the number of servers at node $i$. The traffic intensity (utilization) per node is shown in Table 3 .

For the system to be stable, the traffic intensity $\rho$ must still below one $(\rho<1)$ for each individual node (N1 to N10). Since the aforementioned condition is satisfied for all nodes, the proposed queueing model ensures the system's stability for the long run. 
Table 3 Nodes parameters

\begin{tabular}{llll}
\hline Node number & Service rate (custom./min) & Arrival rate (custom./min) & Traffic intensity \\
\hline $\mathrm{N} 1$ & $\mu_{1}=12$ & $\lambda_{1}=10$ & $\rho_{1}=0.208$ \\
\hline $\mathrm{N} 2$ & $\mu_{2}=10$ & $\lambda_{2}=6.6$ & $\rho_{2}=0.666$ \\
\hline $\mathrm{N} 3$ & $\lambda_{3}=16.6$ & $\rho_{3}=0.666$ \\
\hline $\mathrm{N} 4$ & $\lambda_{3}=25$ & $\lambda_{4}=17.5$ & $\rho_{4}=0.291$ \\
\hline $\mathrm{N} 5$ & $\mu_{4}=3$ & $\lambda_{5}=5.83$ & $\rho_{5}=0.081$ \\
\hline $\mathrm{N} 6$ & $\mu_{5}=12$ & $\iota_{6}=5$ & $\rho_{6}=0.05$ \\
\hline $\mathrm{N} 7$ & $\mu_{6}=4$ & $\lambda_{7}=4.16$ & $\rho_{7}=0.149$ \\
\hline $\mathrm{N} 8$ & $\mu_{7}=2$ & $\lambda_{8}=33.3$ & $\rho_{8}=0.952$ \\
\hline $\mathrm{N} 9$ & $\mu_{8}=35$ & $\iota_{9}=10$ & $\rho_{9}=0.666$ \\
\hline $\mathrm{N} 10$ & $\mu_{9}=15$ & $\lambda_{10}=6.6$ & $\jmath_{10}=0.666$ \\
\hline
\end{tabular}

Next, the steady state probability for each node is calculated $\pi_{n_{i}}$ where $n$ is the number of customers present at node $i$, given that $\pi_{0_{i}}$ represents the idle state (probability of no customer present) at node $i$.

For $\mathrm{M} / \mathrm{M} / \mathrm{c}$ nodes with multiple servers (i.e., N1, N4, $\mathrm{N} 5$, N6 and N7), the steady state probabilities are calculated as below (Equation 13 to 24) [5].

$\pi_{0_{i}}=\left[1+\sum_{i=1}^{c-1} \frac{1}{i !}\left(c \rho_{i}\right)^{i}+\frac{1}{c !}\left(c \rho_{1}\right)^{c} \frac{1}{1-\rho_{i}}\right]^{-1}$

$\pi_{n_{i}}= \begin{cases}\frac{\left(c \rho_{i}\right)^{n_{i}}}{n_{i} !} \pi_{0_{i}}, & n_{i}<c \\ \rho_{i}{ }^{n_{i}} \frac{c^{c}}{c !} \pi_{0_{i}}, & n_{i} \geq c\end{cases}$

For N1:

$\pi_{0_{1}}=\left[1+\sum_{i=1}^{3} \frac{1}{i !}\left(4 \rho_{1}\right)^{i}+\frac{1}{4 !}\left(4 \rho_{1}\right)^{4} \frac{1}{1-\rho_{1}}\right]^{-1}=0.435$

$\pi_{n_{1}}= \begin{cases}\frac{\left(4 \rho_{1}\right)^{n_{1}}}{n_{1} !} \pi_{0_{1}}=\frac{0.832^{n_{1}}}{n_{1} !} 0.435, & n_{1}<4 \\ \rho_{1}{ }^{n_{1}} \frac{4^{4}}{4 !} \pi_{0_{1}}=0.208^{n_{1}} 4.64, & n_{1} \geq 4\end{cases}$

For N4:

$\pi_{0_{4}}=\left[1+\sum_{i=1}^{19} \frac{1}{i !}\left(20 \rho_{4}\right)^{i}+\frac{1}{20 !}\left(20 \rho_{4}\right)^{20} \frac{1}{1-\rho_{4}}\right]^{-1}$

$\pi_{n_{4}}= \begin{cases}\frac{\left(20 \rho_{4}\right)^{n_{4}}}{n_{4} !} \pi_{0_{4}}=\frac{5.82^{n_{4}}}{n_{4} !} 0.003, & n_{4}<20 \\ \rho_{4} n_{4} \frac{20^{20}}{20 !} \pi_{0_{4}}=0.291^{n_{4}} 129703, & n_{4} \geq 20\end{cases}$

For N5:

$\pi_{0_{5}}=\left[1+\sum_{i=1}^{5} \frac{1}{i !}\left(6 \rho_{5}\right)^{i}+\frac{1}{6 !}\left(6 \rho_{5}\right)^{6} \frac{1}{1-\rho_{5}}\right]^{-1}=0.615$

$$
\pi_{n_{5}}= \begin{cases}\frac{\left(6 \rho_{5}\right)^{n_{5}}}{n_{5} !} \pi_{0_{5}}=\frac{0.486^{n_{5}}}{n_{5} !} 0.615, & n_{5}<6 \\ \rho_{5}{ }^{n_{5}} \frac{6^{6}}{6 !} \pi_{0_{5}}=0.081^{n_{5}} \times 39.85, & n_{5} \geq 6\end{cases}
$$

For N6:

$$
\begin{gathered}
\pi_{0_{6}}=\left[\begin{array}{c}
\left.1+\sum_{i=1}^{24} \frac{1}{i !}\left(25 \rho_{6}\right)^{i}+\frac{1}{25 !}\left(25 \rho_{6}\right)^{25} \frac{1}{1-\rho_{6}}\right]^{-1} \\
=0.286
\end{array}\right. \\
\pi_{n_{6}}=\left\{\begin{array}{c}
\frac{\left(25 \rho_{6}\right)^{n_{6}}}{n_{6} !} \pi_{0_{6}}=\frac{1.25^{n_{6}}}{n_{6} !} 0.286, n_{6}<25 \\
\rho_{6}{ }^{n_{6}} \frac{25^{25}}{25 !} \pi_{0_{6}}=0.05^{n_{6}} \times 16 \times 10^{8}, n_{6} \geq 25
\end{array}\right.
\end{gathered}
$$

For N7:

$$
\begin{gathered}
\pi_{0_{7}}=\left[\begin{array}{c}
\left.1+\sum_{i=1}^{13} \frac{1}{i !}\left(14 \rho_{7}\right)^{i}+\frac{1}{14 !}\left(14 \rho_{7}\right)^{14} \frac{1}{1-\rho_{7}}\right]^{-1} \\
=0.124
\end{array}\right. \\
\pi_{n_{7}}=\left\{\begin{array}{cc}
\frac{\left(14 \rho_{7}\right)^{n_{7}}}{n_{7} !} \pi_{0_{7}}=\frac{2.086^{n_{7}}}{n_{7} !} 0.124, & n_{7}<14 \\
\rho_{7}{ }^{n_{7}} \frac{14^{14}}{14 !} \pi_{0_{7}}=0.149^{n_{7}} \times 15828, & n_{7} \geq 14
\end{array}\right.
\end{gathered}
$$

For M/M/1 nodes (i.e., N2 and N10), the steady state probabilities are calculated as below (Equation 25 to 28).

For N2:

$\pi_{0_{2}}=1-\rho_{2}=0.334$

$\pi_{n_{2}}=\left(1-\rho_{2}\right) \cdot \rho_{2}{ }^{n_{2}}=0.334\left(0.666^{n_{2}}\right)$

For N10:

$\pi_{0_{10}}=1-\rho_{10}=0.334$

$\pi_{n_{10}}=\left(1-\rho_{10}\right) \cdot \rho_{10}{ }^{n_{10}}=0.334\left(0.666^{n_{10}}\right)$ 
For the rest of network nodes (i.e., N3, N8 and N9), they are $\mathrm{M} / \mathrm{M} / \infty$ with steady state probabilities calculated as below (Equation 29 to 34) [4].

For N3:

$\pi_{0_{3}}=e^{-\rho_{3}}=0.513$

$\pi_{n_{3}}=\frac{\rho_{3}^{n_{3}}}{n_{3} !} e^{-\rho_{3}}=0.513 \frac{0.666^{n_{3}}}{n_{3} !}$

For N8:

$\pi_{0_{8}}=e^{-\rho_{8}}=0.386$

$\pi_{n_{8}}=\frac{\rho_{8}^{n_{8}}}{n_{8} !} e^{-\rho_{8}}=0.386 \frac{0.952^{n_{8}}}{n_{8} !}$

For N9:

$\pi_{0_{9}}=e^{-\rho_{9}}=0.513$

$\pi_{n_{9}}=\frac{\rho_{9}^{n_{9}}}{n_{9} !} e^{-\rho_{9}}=0.513 \frac{0.666^{n_{9}}}{n_{9} !}$

Now, for the overall system's steady state probability can be calculated as Equation 35:

$$
\pi_{n_{1}: n_{10}}=\pi_{n_{1}} \cdot \pi_{n_{2}} \cdot \pi_{n_{3}} \cdot \pi_{n_{4}} \cdot \pi_{n_{5}} \cdot \pi_{n_{6}} \cdot \pi_{n_{7}} \cdot \pi_{n_{8}} \cdot \pi_{n_{9}} \cdot \pi_{n_{10}}
$$

\section{Results}

In this section, the results of the proposed queueing model are discussed in terms of different performance measures such as expected number of customers in network nodes, queue lengths, mean system waiting time and queueing time.

Firstly, to check the steady state probability of having no customer in the network (i.e., the idle steady state), we substitute $\pi_{0_{i}}$, calculated earlier, in Equation 35 to yield (Equation 36):

$$
\begin{gathered}
\pi_{0}=\pi_{0_{1}} \cdot \pi_{0_{2}} \cdot \pi_{0_{3}} \cdot \pi_{0_{4}} \cdot \pi_{0_{5}} \cdot \pi_{0_{6}} \cdot \pi_{0_{7}} \cdot \pi_{0_{8}} \cdot \pi_{0_{9}} \cdot \pi_{0_{10}} \\
=3.22 \times 10^{-7}
\end{gathered}
$$

Next, another performance measure is determined which is the mean number of customers in the network, given as Equation 37:

$$
\begin{gathered}
L=L_{1}+L_{2}+L_{3}+L_{4}+L_{5}+L_{6}+L_{7}+L_{8}+L_{9} \\
+L_{10}
\end{gathered}
$$

where $L_{1}, L_{2}, \ldots L_{10}$ represent the average number of customers at N1, N2, ...N10 respectively. The value of $L$ at each node depends on its number of servers. For N1, N4, N5, N6 and N7 (M/M/c) (Equation 38): $L=E[N]=\sum_{n=0}^{\infty} n \pi_{n}=L_{q}+c \rho$

where $L_{q}$ represents the average number of customers waiting in queue (queue length) at each node. Since the queue starts build up after $c$ customers, the average queue length is given as (Equation 39):

$L_{q}=E[N]=\sum_{n=c}^{\infty}(n-c) \pi_{n}$

Equation 14 is substituted in Equation 39 to yield (Equation 40):

$L_{q}=\sum_{n=c}^{\infty}(n-c) \rho^{n} \frac{c^{c}}{c !} \pi_{0}=\sum_{n=c}^{\infty} n \rho^{n} \frac{c^{c}}{c !} \pi_{0}$

$\sum_{n=c}^{\infty} c \rho^{n} \frac{c^{c}}{c !} \pi_{0}$

By using derivatives of geometric series stated in [20], Equation 40 becomes as Equation 41:

$L_{q}=\frac{(c \rho)^{c}}{c !} \frac{\rho}{(1-\rho)^{2}} \pi_{0}$

By substituting Equation 41 in Equation 38, it yields (Equation 42):

$L=\frac{(c \rho)^{c}}{c !} \frac{\rho}{(1-\rho)^{2}} \pi_{0}+c \rho$

Now, the average number of customers at each node is calculated using Equation 42 as the following:

$L_{1}=\frac{\left(4 \rho_{1}\right)^{4}}{4 !} \frac{\rho_{1}}{\left(1-\rho_{1}\right)^{2}} \pi_{0_{1}}+4 \rho_{1}=0.835$ customer

$L_{4}=\frac{\left(20 \rho_{4}\right)^{20}}{20 !} \frac{\rho_{4}}{\left(1-\rho_{4}\right)^{2}} \pi_{04}+20 \rho_{4}=5.82$ customers

$L_{5}=\frac{\left(6 \rho_{5}\right)^{6}}{6 !} \frac{\rho_{5}}{\left(1-\rho_{5}\right)^{2}} \pi_{0_{5}}+6 \rho_{5}=0.49$ customer

$L_{6}=\frac{\left(25 \rho_{6}\right)^{25}}{25 !} \frac{\rho_{6}}{\left(1-\rho_{6}\right)^{2}} \pi_{0_{6}}+25 \rho_{6}=1.25$ customers

$L_{7}=\frac{\left(14 \rho_{7}\right)^{14}}{14 !} \frac{\rho_{7}}{\left(1-\rho_{7}\right)^{2}} \pi_{0}+14 \rho_{7}=2.09$ customers

For N3, N8 and N9 (M/M/o):

In $\mathrm{M} / \mathrm{M} / \infty$, the mean number of customers present must be equal to $\lambda$ times the mean service time $1 / \mu$ which reveals that $L=\rho$ that results in:

$L_{3}=L_{9}=\rho_{3}=\rho_{9}=0.666$ customer

$L_{8}=\rho_{8}=0.952$ customer

For N2 and N10 (M/M/1):

$L_{2}=L_{10}=\frac{\rho_{2}}{1-\rho_{2}}=\frac{\rho_{10}}{1-\rho_{10}}=1.994$ customers

After all queue lengths have been determined (i.e., $L_{1}$ to $L_{10}$ ), the average number of customers in the whole system/network is calculated using Equation 37 to yield:

$L=16.76$ customers

Another performance measure to determine is the mean time a customer takes in the network. According to Little's law, this mean system time is simply the ratio between the system queue length to the sum of external arrivals to the system as following:

$W=\frac{L}{\lambda_{\text {in } 1}+\lambda_{\text {in } 2}}=\frac{16.76}{10+6.66}=1.006 \mathrm{~min}$ 
Hence, each customer takes approximately one minute in the system which is the summation of both waiting time and serving time. In order to determine the average queueing time (i.e., waiting time) each customer consumes in the system, the following formula (Equation 43) is applied:

$W_{q}=\frac{\sum_{i=1}^{10} L_{q_{i}}}{\lambda_{i n 1}+\lambda_{i n 2}}$

For M/M/c nodes, Equation 41 is used to calculate $L_{q}$ to reveal:

$L_{q_{1}}=0.003$ and $L_{q_{4}}=L_{q_{5}}=L_{q_{6}}=L_{q_{7}}=0$

For the $\mathrm{M} / \mathrm{M} / \infty^{\infty}$ nodes, there is no waiting. Hence, $L_{q_{3}}=L_{q_{8}}=L_{q_{9}}=0$.

For $\mathrm{M} / \mathrm{M} / 1$ nodes, the queue lengths are determined as:

$L_{q_{2}}=L_{q_{10}}=\frac{\rho_{2}^{2}}{1-\rho_{2}}=\frac{\rho_{10}^{2}}{1-\rho_{10}}=1.33$ customers

Then, the values of $L_{q_{1}}$ to $L_{q_{10}}$ are substituted in Equation 43 to yield:

$W_{q}=\frac{2.6696}{10+6.66}=0.16 \mathrm{~min}$

\section{Discussion}

It is evident that most of the time the customers spend at the serving stations to get served for a mean of $0.846 \mathrm{~min}$ that is compared to only $0.16 \mathrm{~min}$ waiting for getting their service, so that the total time spent in the system is 1.006 minutes. Figure 3 depicts the highest percentage involves the serving phase at $84-85 \%$ compared to the waiting phase at $15-16 \%$. In other words, the customers are getting services, $85 \%$ (yellowish in Figure 3) of their time inside the system, whereas they only wait for $15 \%$ (greenish in Figure 3) of their time. Furthermore, when the system has $84 \%$ of the total number of its customers getting served, it experiences only $16 \%$ of them standing in queues. That is an explicit indication that the system is stable due to low arrival rates in contrast with no-pandemic life.

The mean number of customers and queue length at each node is summarized in Figure 4. The figure shows that the busiest node in the network is N4 with almost 6 customers are existing. However, that does not indicate the N4 is a bottleneck since it has reasonably acceptable traffic intensity rate at $\rho=$ 0.291 due to the existence of 20 servers. On the other hand, only 0.5 customer appear as average at N5 which records the quietest node. Furthermore, N2 and $\mathrm{N} 10$ are the only nodes in the network with customers have to wait in queues before getting served due to having only a single server each.

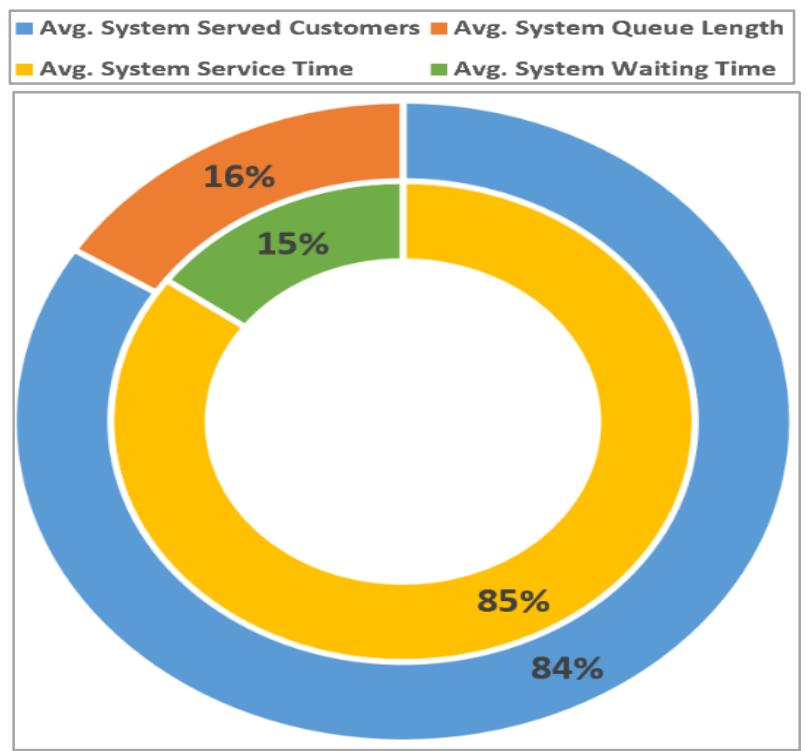

Figure 3 Percentage chart of system performance measures

Consequently, the different performance measures presented in this section are validating the analytical approach derived in sections 4 for the proposed network state diagram structures in Figure 2. That is because the system can reach steady state (stability) and serve customers with an almost 1 minute as an average which is fairly acceptable. Moreover, the network nodes are not dealing with long queues which means that the customers are getting served most of time with minimum waiting. Furthermore, in case of witnessing an increase in shopper rate, which reflects on queue lengths, the analytical approach followed in the previous section can be re-planned to cope with each node's requirement. For example, maximizing the number of servers at N2 and N10 with minimizing it at $\mathrm{N} 5$, then re-calculations are made in the same analytical workflow.

However, the proposed system has some limitations. In terms of limitations, the proposed queueing system will suffer once the pandemic consequences are mitigated worldwide. This mitigation will attract more customers to visit shopping malls which in turns degrades system's stability unless re-planning is adopted based on the same analytical workflow pursued in this study. This implication may emerge from the proposed design is presented for this particular pandemic era with only 16.6 customers/ min arrivals to the mall, whereas a huge increase in arrivals will be an explicit performance limitation. 
Also, from the analysis of the system, it is noticeable that N8 (i.e., corridor node) is almost fully utilized at $\rho=0.952$. This particular node is considered a critical node that may degrade the whole network once the arrivals increase without raising the number of servers. This node is a system bottleneck that possibly be overcome via implanted other nodes to balance the arrival loads, but that at the expense of overall system complexity. Moreover, the failure in a certain node of the network causes its inbound transition probability on Figure 2 to be handled by other nodes. For example, if N4 fails, then its inbound 0.45 transition probability must be distributed on N5, N6, N7 and N8 (as the sum of N3 outbound probabilities is 1) which adds extra intensive traffic on them to process. As a result, the bottleneck N8 causes system failure if its $\rho$ exceeds 1. Furthermore, the customers in the current system consume more of their time inside the system in service with less than 10 seconds waiting whereas this waiting time will be multiples once the traffic intensities and queue lengths increase. Moreover, as queue lengths getting longer, another limitation emerges in the system that is related to customer and server behaviors. Unlike the usual behaviors assumed in this system, customers may behave unusually such as join the queue but then renege or jockey (i.e., jumping among queues) and that requires alternative design considerations.

Moreover, although it is true that the analytical workflow approach presented in this paper can be generalized, that is particularly reasonable in a similar life queue. In other words, the design assumptions may be amended, tailored or even extended to other queue systems to suit their particular requirements. One last limitation is the infrastructural survey of the mall's building. For security precautions, the mall's administration did not give permissions to reveal some general statistics about the mall such as the average number of arrivals, departures or the number of checkout stations in the mall's facilities. In consequence, human observation was the applicable mean to record data by visiting the network nodes inperson. A complete list of abbreviations is shown in Appendix I.

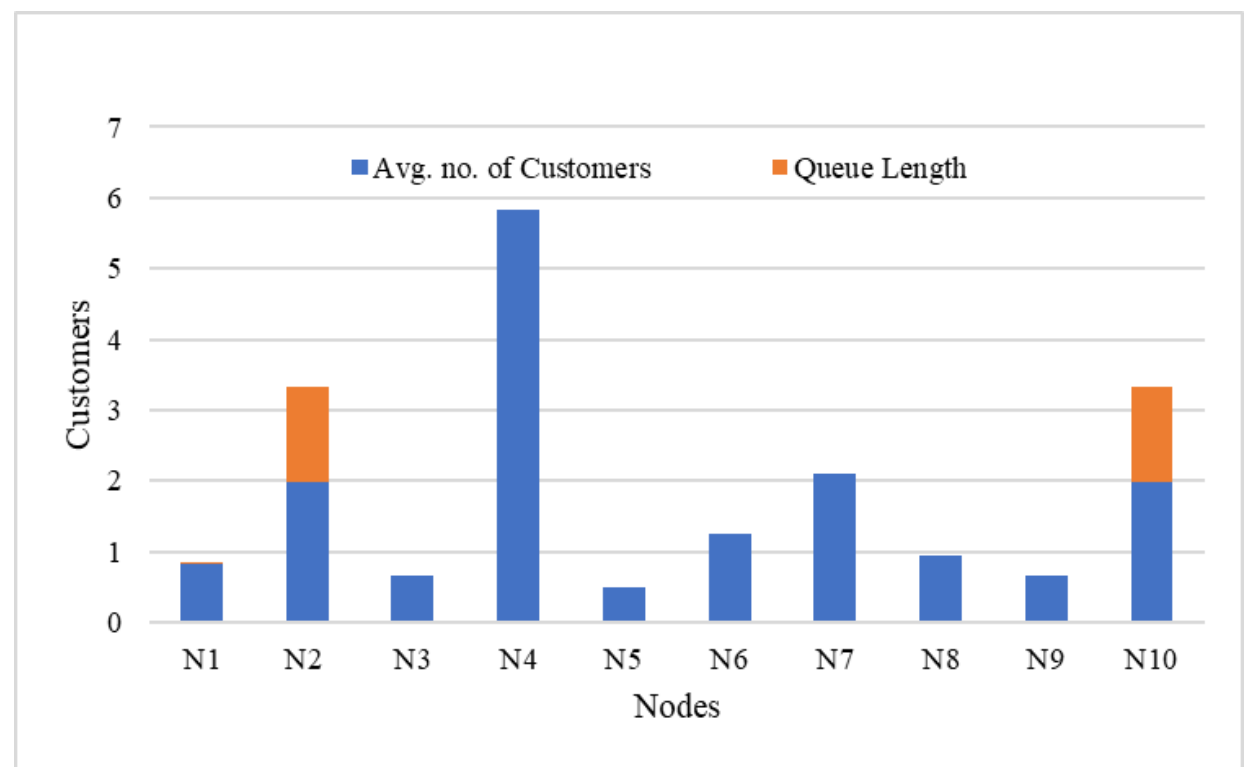

Figure 4 A summary of L and Lq at each node

\section{Conclusion and future work}

In this research, an analytical queueing model has been proposed to study the impacts of COVID-19 on the shoppers' popularity and how the proposed system performs with mall customer arrivals. The system model consists of an open network of 10 nodes that represent entrance gates, fast-food restaurants, hypermarket, clothing retail outlets, exchange offices, a corridor and exits. Each node is modelled with an appropriate model queue type depending on the real equivalent servers of the mall. The analysis of the proposed system is investigated based on the recorded data in terms of arrival and service rates of each individual serving node. The system model shows a stability in the long run based on mathematical expressions of the traffic intensity of 
each node which revealed $\rho<1$. However, N8 is the busiest node in the network as its traffic intensity is 0.952 which approaches fully utilization. Also, the steady state probability $\pi \_\left(n \_i \text { )is presented for } n\right.$ number of customers present at node i with network idle steady state probability of $\llbracket 3.22 \times 10 \rrbracket^{\wedge}(-7)$. Moreover, the results show that the average number of customers in the network is 16.76 with only 2.66 customers waiting in queues. Furthermore, the mean time a customer spends in queue is 0.16 minute from a total time spend in the whole network of 1.006 minute.

In consequence, it is noticeable that the number of arrivals to the mall is still with low rate and the majority of time the customers spend is for service with very short time for waiting due to current COVID-19 pandemic consequences. In other words, the values of $\lambda i \mathrm{i}$ and $\mu \mathrm{i}$ will definitely alter in the future if normal social life resumes, and that surely pushes for an increase in arrivals and consequently a growth in average number of customers in queues with more waiting time. Since then, techniques for reducing and prediction of waiting time [21, 22] as well as alternative queue scheduling and priority mechanisms [23, 24] are all essential to maintain system stability. Moreover, in case that COVID-19 mitigates its effects on social life, other queueing issues related to customer behavior will emerge. For example, balking and reneging queues as well as double-sided queues with patient and impatient visitors [25] will be crucial to consider.

\section{Acknowledgment}

None.

\section{Conflicts of interest}

The authors have no conflicts of interest to declare.

\section{References}

[1] Alfa AS. Applied discrete-time queues. Springer New York; 2016.

[2] Giambene G. Queuing theory and telecommunications networks and applications, 2005 springer science+ business media. New York, USA: Springer; 2014.

[3] PalunčIć F, Alfa AS, Maharaj BT, Tsimba HM. Queueing models for cognitive radio networks: a survey. IEEE Access. 2018; 6:50801-23.

[4] Smith JM. Introduction to queueing networks: theory $\cap$ practice. Springer; 2018.

[5] Shortle JF, Thompson JM, Gross D, Harris CM. Fundamentals of queueing theory. John Wiley \& Sons; 2018.

[6] Jhala N, Bhathawala P. Analysis and application of queuing theory in supermarkets. International Journal of Innovative Research in Science, Engineering and Technology. 2017; 6(9):17974-9.

[7] Igwe A, Onwuere JU, Egbo OP. Efficient queue management in supermarkets: a case study of makurdi town, Nigeria. European Journal of Business and Management. 2014; 6(39):185-92.

[8] Priyangika JS, Cooray TM. Analysis of the sales checkout operation in supermarket using queuing theory. Universal Journal of Management. 2016.

[9] Prasad V, Vh B, Koka TA. Mathematical analysis of single queue multi server and multi queue multi server queuing models: comparison study. Global Journal of Mathematical Analysis. 2015; 3(3):97-104.

[10] Chinwuko EC, Daniel EC, Ugochukwu OP, OO J. Analysis of a queuing system in an organization (a case study of first bank PLC, Nigeria). American Journal of Engineering Research. 2014; 3(2):63-72.

[11] Sharma A, Barua PB. Application of queuing theory in a small enterprise. International Journal of Engineering Trends and Technology. 2015; 27(2):10510.

[12] Majedkan NA, Idrees BA, Ahmed OM, Haji LM, Dino HI. Queuing theory model of expected waiting time for fast diagnosis nCovid-19: a case study. In international conference on advanced science and engineering 2020 (pp. 127-32). IEEE.

[13] Chatterjee K, Chatterjee K, Kumar A, Shankar S. Healthcare impact of COVID-19 epidemic in India: a stochastic mathematical model. Medical Journal Armed Forces India. 2020; 76(2):147-55.

[14] Wang CH. A three-level health inspection queue based on risk screening management mechanism for postCOVID global economic recovery. IEEE Access. 2020; 8:177604-14.

[15] Rezzouki M, Ouajih S, Ferré G. Monitoring social distancing in queues using infrared array sensor. IEEE Sensors Journal. 2021.

[16] Tyagi M, Tyagi PK, Singh S, Sathpathy S, Kant S, Gupta SK, et al. Impact of application of queuing theory on operational efficiency of patient registration. Medical Journal Armed Forces India. 2021.

[17] Jen GH, Chen SY, Chang WJ, Chen CN, Yen AM, Chang RE. Evaluating medical capacity for hospitalization and intensive care unit of COVID-19: a queue model approach. Journal of the Formosan Medical Association. 2021; 120:86-94.

[18] Thirupathieswaran R, Prakash CS, Krishnan RS, Narayanan KL, Kumar MA, Robinson YH. Zero queue maintenance system using smart medi care application for Covid-19 pandemic situation. In third international conference on intelligent communication technologies and virtual mobile networks 2021 (pp. 1068-75). IEEE.

[19] Soman S, Rai S, Ranjan P, Cheema AS, Srivastava PK. Mobile-augmented smart queue management system for hospitals. In 33rd international symposium on computer-based medical systems 2020 (pp. 421-6). IEEE.

[20] Stewart WJ. Probability, Markov chains, queues, and simulation. Princeton University Press; 2009. 
[21] Aung T, Naing LL. Comparative study on different queuing models to reduce waiting time in brahmaso clinic. International Journal of Scientific and Research Publications. 2019; 9(6).

[22] Stagge A. A time series forecasting approach for queue wait-time prediction. Digitala Vetenskapliga Arkivet. 2020.

[23] Dey NS, Gunasekhar T. A comprehensive survey of load balancing strategies using hadoop queue scheduling and virtual machine migration. IEEE Access. 2019; 7:92259-84.

[24] Eiada HA. Improve the performance of the fuzzy priority queue in baghdad teaching hospital. AlRafidain University College for Sciences. 2018:20631.

[25] Diamant A, Baron O. Double-sided matching queues: priority and impatient customers. Operations Research Letters. 2019; 47(3):219-24.

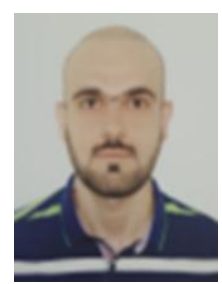

Aymen A. Al-Kadhimi attained his MSc degree in communications and signal processing from the University of Leeds, UK in 2015. He is currently a researcher at the Computer Networks Engineering department, Al-Nahrain University in Baghdad, Iraq. His research interests are around Modern Wireless Communications Technologies and Signals Processing Techniques in which he has several papers published in conferences and Scopus-indexed journals. Along with lecturing to BSc students, he is currently pursuing his $\mathrm{PhD}$ study in information and communication engineering.

Email: aymen.mohammed@ nahrainuniv.edu.iq

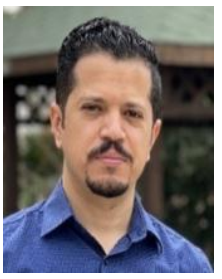

Mustafa Abdulkadhim is a lecturer in the computer networks engineering department, he is holding a master's degree from the University of Pune India. He published several papers in the field of IoT and SDN and Computer Networking Technologies. Also working as the manager for the Cisco networking academy and the continues education center in Al-Nahrain university.

Email:mstfkadum@nahrainuniv.edu.iq

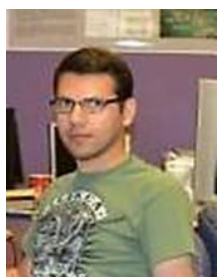

Salim A. Mohammed Ali is an assistant lecturer at the department of Computer Networks Engineering at AlNahrain University in Baghdad, Iraq. He has a master's degree in Computer Engineering from Clemson University, SC, USA. Currently, he is a PhD candidate in Information and Communication Engineering. His research interests are focused on Heterogeneous Computing and Network Applications.

Email: salimm@ieee.org

\begin{tabular}{lll}
\multicolumn{2}{l}{ Appendix I } \\
\hline S. No. & Abbreviation & Description \\
\hline 1 & COVID-19 & Coronavirus disease of 2019 \\
\hline 2 & D & Deterministic \\
\hline 3 & E & Erlang \\
\hline 4 & FCFS & First come First Served \\
\hline 5 & G & General \\
\hline 6 & H & Hyper-exponential \\
\hline 7 & ICU & Intensive Care Unit \\
\hline 8 & M & Markovian \\
\hline 9 & SDN & Software Defined Network \\
\hline & & \\
\hline
\end{tabular}

